\title{
FLAME SURFACE DENSITIES IN PREMIXED COMBUSTION AT MEDIUM TO HIGH TURBULENCE INTENSITIES
}

\author{
ÖMER L. GÜLDER* \\ Institute for Aerospace Studies, University of Toronto, \\ Toronto, Ontario, Canada
}

GREGORY J. SMALLWOOD

National Research Council Canada, Ottawa,
Ontario, Canada

The surface densities of flame fronts in turbulent premixed propane/ air flames were determined experimentally. The instantaneous flame fronts were visualized using laser induced fluorescence (LIF) of $\mathrm{OH}$ on two Bunsen type burners of 11.2 and $22.4 \mathrm{~mm}$ diameters. Nondimensional turbulence intensity, $u^{\prime} / S_{\mathrm{L}}$, was varied from 0.84 to 15 , and the Reynolds number, based on the integral length scale, varied from 34 to 467 . These flames are in the flamelet combustion regime as defined by the most recent turbulent premixed combustion diagrams. From 100 to 800 images were recorded for each experimental condition. Flame surface densities were obtained from the instantaneous maps of the progress variable, which is zero in the reactants and unity in the products. These flame surface densities were corrected for the mean direction cosines of the flame fronts, which had a typical value of 0.69 for the Bunsen flames. In the non-dimensional turbulence intensity range of up to 15 , it was found that the maximum flame surface density and the integrated flame surface density across the flame brush do not show any significant dependence on turbulence intensity. This was discussed in the framework of a flame surface density-based turbulent premixed flame propagation closure model.

Received 20 June 2005; accepted 13 January 2006.

*Address correspondence to ogulder@utias.utoronto.ca 
The implication is that the conceptual increase in flame surface density with turbulence may not be the dominant mechanism for flame velocity enhancement in turbulent combustion in the region specified as the flamelet combustion regime by the current turbulent premixed combustion diagrams. Small-scale transport of heat and species may be more important and chemistry may not be decoupled from turbulence. Further, the applicability of the flamelet approach may be limited to a much smaller range of conditions than presently believed.

Keywords: flamelets, flame surface density, turbulent premixed combustion

\section{INTRODUCTION}

Although the structure of turbulent premixed flames, i.e. either flamelet or thickened flame sheet, still is a continuing matter of debate (e.g., Ronney and Yakhot, 1992; Zimont, 2000; Kortschik et al. 2004), the widely held belief is that the structure (chemical and hydrodynamic) of a stretched laminar premixed flame can be preserved in a turbulent flow field over a range of conditions collectively known as the flamelet regime. In this regime, the turbulent flame is considered as a propagating sheet composed of stretched flamelets of small but finite thickness (Bray, 1990; Cant and Bray, 1988; Peters, 1999). Since the instantaneous behavior of these asymptotically thin layers is considered the same as those of laminar flames, the turbulent burning velocity can be approximated by the product of the flamelets' surface area and the laminar burning velocity corrected for the effect of stretch and flame curvature (Bray and Cant, 1991). In this way, the laminar flamelet assumption is exploited to avoid dealing with the complex chemical structure of the turbulent flame. Thus, the flamelet assumption asserts that the flame front behaves as a constant-property passive scalar surface. Most recent experimental and numerical studies imply that the flamelet combustion regime, where the flamelet approach can be used, is much wider than previously thought (Bédat and Cheng, 1995; Peters, 1999; Poinsot et al., 1990; Roberts et al., 1993).

Within the laminar flamelets regime, the complex chemical kinetics mechanism is represented in terms of the laminar flame propagation velocity, $S_{\mathrm{L}}$. The wrinkling of the flame front surface by turbulence is described by the mean flame-surface area per unit volume that is 
known as the flame surface density, $\Sigma$. The mean rate of conversion of reactants into products per unit volume, $\langle\dot{\omega}\rangle$, can be expressed as

$$
\langle\dot{\omega}\rangle(\boldsymbol{x})=\rho_{\mathrm{u}} S_{\mathrm{d}} \Sigma(\boldsymbol{x})
$$

where $\rho_{\mathbf{u}}$ is the density of reactants, $\Sigma(x)$ is the local flame surface density, and $S_{\mathrm{d}}$ is the local propagation velocity of the flamelets. $S_{\mathrm{d}}$ was defined as the reactant consumption velocity by Bray and Cant (1991), but also called as the flamelet displacement velocity (see, e.g., Sinibaldi et al., 1998). The ratio of the laminar burning velocity of a planar and unstretched flamelet, $S_{\mathrm{L}}$, to the flamelet displacement velocity, $S_{\mathrm{d}}$, is dependent on the flame curvature and the aerodynamic strain rate.

Simple algebraic closure models have been proposed for flame surface density, $\Sigma$ (Bray, 1980; Bray et al., 1985). The Bray-Moss-Libby model (Bray et al., 1985) for $\Sigma$ is based on the spatial distribution of flame crossings along a contour of mean progress variable, $\langle c\rangle$, which is 0 in the reactants and 1 in the products. An alternative formulation for modeling the spatial variation of the flame surface density, based on the gradient of the progress variable across the flame front (Pope, 1988), is

$$
\Sigma(x) \equiv\left\langle\Sigma^{\prime}(x)\right\rangle=\left\langle|\nabla c| \delta\left(c-c_{f}\right)\right\rangle
$$

where $\nabla c$ is the spatial flame front gradient, $\delta\left(c-c_{f}\right)$ is the instantaneous flame front position ( $\delta$ is the Kronecker delta), and $\Sigma^{\prime}(x)$ is the instantaneous local flame surface density.

In flamelet models, the flame surface density is obtained either using an algebraic closure or by way of a godelin transport equation, known as the $\Sigma$-equation. The $\Sigma$-equation was first formulated by Marble and Broadwell (1977). In a turbulent flow field, the averaged $\Sigma$-equation contains terms representing transport by mean flow, transport by turbulence and flame propagation, and production and destruction by flame stretch of the flame surface. Modelling assumptions are needed for the transport due to flame propagation, the turbulent diffusion velocity, and turbulent flame stretch. Unfortunately, these terms are not experimentally accessible. Further discussion on the $\Sigma$-equation and its numerical solution schemes can be found in Pope (1988), Trouvé and Poinsot (1994), Boger et al. (1998), and Prasad et al. (1999).

Most of the experimental data on flame surface density are limited to low turbulence intensities, e.g., Deschamps et al. (1994), Deschamps et al. (1996), Shepherd (1996), and Ghenaï (1996). In most of studies, the threedimensional information has been extracted from the two-dimensional 
data by making certain assumptions. In Deschamps et al. (1996), images from orthogonal planes have been used to determine the mean orientation angle, i.e., the direction cosine, along the line of intersection of the planes. Several studies concluded that in a wide range of flame geometries, the mean direction cosine is about 0.65-0.75 (Deschamps, 1996; Shepherd and Ashurst, 1992; Zhang et al., 1998). Bingham et al. (1998) introduced a new technique, crossed-plane tomography, for direct determination of the instantaneous flamelet surface normals by using data from tomographic images recorded simultaneously from two orthogonal laser illumination planes, which were used to determine the flame surface density (Bingham et al., 1998; Knaus et al., 1999).

Experimental data obtained in Bunsen flames and firing homogenous charge spark ignition engines at low turbulence intensities, i.e., $u^{\prime} / S_{\mathrm{L}}$ less than 2, did not show any significant variation of the flame surface density with turbulence intensity (Deschamps et al., 1994, 1996; Smallwood and Deschamps, 1996; Knaus et al., 1999). To provide experimental flame surface density data at medium to high turbulence intensities, we have carried out an experimental program and determined the flame surface densities of turbulent premixed flames on a Bunsen type burner. The Reynolds numbers, $\operatorname{Re}_{\Lambda}$, based on the integral length scale, $\Lambda$, cover the range 34 to 467 , and $u^{\prime} / S_{\mathrm{L}}$ covers the range from 0.84 to 15 .

\section{EXPERIMENTAL METHODOLOGY}

The turbulent premixed conical flames studied were produced by two axisymmetric Bunsen-type burners with inner nozzle diameters of 11.2 and $22.4 \mathrm{~mm}$. Low turbulence intensity flames studied on the $22.4 \mathrm{~mm}$ diameter burner were reported previously (Deschamps et al., 1996). Premixed turbulent propane-air flames with equivalence ratios of 0.8 and 1.0 were stabilized by using an annular propane pilot for low turbulence flames and a hydrogen pilot for high turbulence ones. Turbulence levels were controlled by perforated plates positioned three nozzle diameters upstream of the burner exit. The turbulence parameters were measured under reacting conditions by LDV of fine silicone oil droplets seeded in the flow. The integral length scales, $\Lambda$, and turbulence intensities, $u^{\prime}$, reported in Table 1 , were measured on the burner centerline at the nozzle exit.

The integral length scale was determined using Taylor's hypothesis. The turbulence intensity field measured for the flame condition " $M$ " is 
Table 1. Summary of experimental conditions and results

\begin{tabular}{lrlllllllll}
\hline $\begin{array}{l}\text { Data } \\
\text { Set }\end{array}$ & $d(\mathrm{~mm})$ & $\Phi$ & $U(\mathrm{~m} / \mathrm{s})$ & $\Lambda(\mathrm{mm})$ & $u^{\prime}(\mathrm{m} / \mathrm{s})$ & $u^{\prime} / S_{\mathrm{L}}$ & $\Lambda / \delta_{\mathrm{L}}$ & $\delta_{\mathrm{T}}(\mathrm{mm})$ & $\Sigma_{\max }(\mathrm{mm})-1$ & $S_{\mathrm{T}} / S_{\mathrm{L}}$ \\
\hline A & 22.4 & 1.0 & 11 & 1.5 & 0.36 & 0.84 & 40 & 48 & 0.3 & 3.1 \\
B & 22.4 & 1.0 & 14 & 2.3 & 0.6 & 1.4 & 62 & 47 & 0.31 & 4.5 \\
C & 22.4 & 0.8 & 14 & 2.5 & 0.6 & 2.0 & 47 & 57 & 0.3 & 5.8 \\
D & 11.2 & 1.0 & 39 & 1.5 & 2.3 & 5.3 & 40 & 64 & 0.28 & 8.9 \\
E & 11.2 & 1.0 & 42 & 1.8 & 2.8 & 6.5 & 48 & 51 & 0.37 & 7.4 \\
F & 11.2 & 1.0 & 42 & 1.8 & 2.8 & 6.5 & 48 & 43 & 0.31 & 7.8 \\
G & 11.2 & 1.0 & 53 & 1.6 & 3.1 & 7.1 & 43 & 55 & 0.24 & 6.8 \\
H & 11.2 & 1.0 & 56 & 1.6 & 3.4 & 7.9 & 43 & 58 & 0.27 & 9.2 \\
I & 11.2 & 1.0 & 73 & 1.8 & 3.7 & 8.6 & 48 & 64 & 0.25 & 10.8 \\
J & 11.2 & 0.8 & 42 & 1.9 & 2.7 & 9.1 & 36 & 59 & 0.36 & 8.8 \\
K & 11.2 & 1.0 & 77 & 1.6 & 4.5 & 10.4 & 43 & 56 & 0.33 & 11.7 \\
L & 11.2 & 0.8 & 72 & 1.7 & 3.9 & 12.9 & 32 & 58 & 0.22 & 13.5 \\
M & 11.2 & 0.8 & 77 & 1.7 & 4.5 & 15 & 32 & 66 & 0.31 & 19.5 \\
\hline
\end{tabular}

Symbols: $d=$ burner diameter; $\Phi=$ fuel-air equivalence ratio; $U=$ mean flow velocity; $\Lambda=$ integral length scale; $u^{\prime}=$ rms velocity fluctuation; $u^{\prime} / S_{\mathrm{L}}=$ non-dimensional turbulence intensity; $\Lambda / \delta_{\mathrm{L}}=$ ratio of integral length scale to laminar flame thickness $\left(\delta_{\mathrm{L}}=\nu / S_{\mathrm{L}}\right.$, where $\nu$ is the kinematic viscosity); $\delta_{\mathrm{T}}=$ maximum flame brush thickness; $\Sigma_{\max }=$ maximum flame surface density; $S_{\mathrm{T}} / S_{\mathrm{L}}=$ ratio of turbulent to laminar burning velocity.

shown in Figure 1. Similar profiles were obtained for other flame conditions listed in Table 1.

The instantaneous flame fronts were visualized by laser induced fluorescence of $\mathrm{OH}$. A tunable excimer laser was wavelength tuned to a strong $\mathrm{OH}$ radical molecular resonance line, $\mathrm{Q}_{1}(3)$, which lies within the $A^{2} \Sigma^{+}\left(v^{\prime}=0\right) \leftarrow X^{2} \Pi\left(v^{\prime \prime}=0\right)$ electronic band. The dimensions of the laser sheet at the burner centerline were about $17 \mathrm{~cm}$ by $100 \mu \mathrm{m}$ (FWHM) in the vertical and horizontal planes, respectively. The sheet thickness was less than $150 \mu \mathrm{m}$ over the full flame width. The sheet optics consisted of a cylindrical lens for vertical plane expansion and a spherical lens for horizontal plane focusing. For LIF of OH, the images were acquired with an intensified CCD detector $(576 \times 384$ pixels $)$ giving a flame image spatial resolution of $150 \mu \mathrm{m}$. This resolution was much finer than the inner cutoff for these flames, and thus all scales of wrinkling were captured in the images (Deschamps et al., 1996). All LIF images included full views of the flame from burner exit to flame tip.

The images were then systematically binarized by setting a threshold. Pixel values of 1 indicate a progress variable equal to 1 (burnt gases) and 


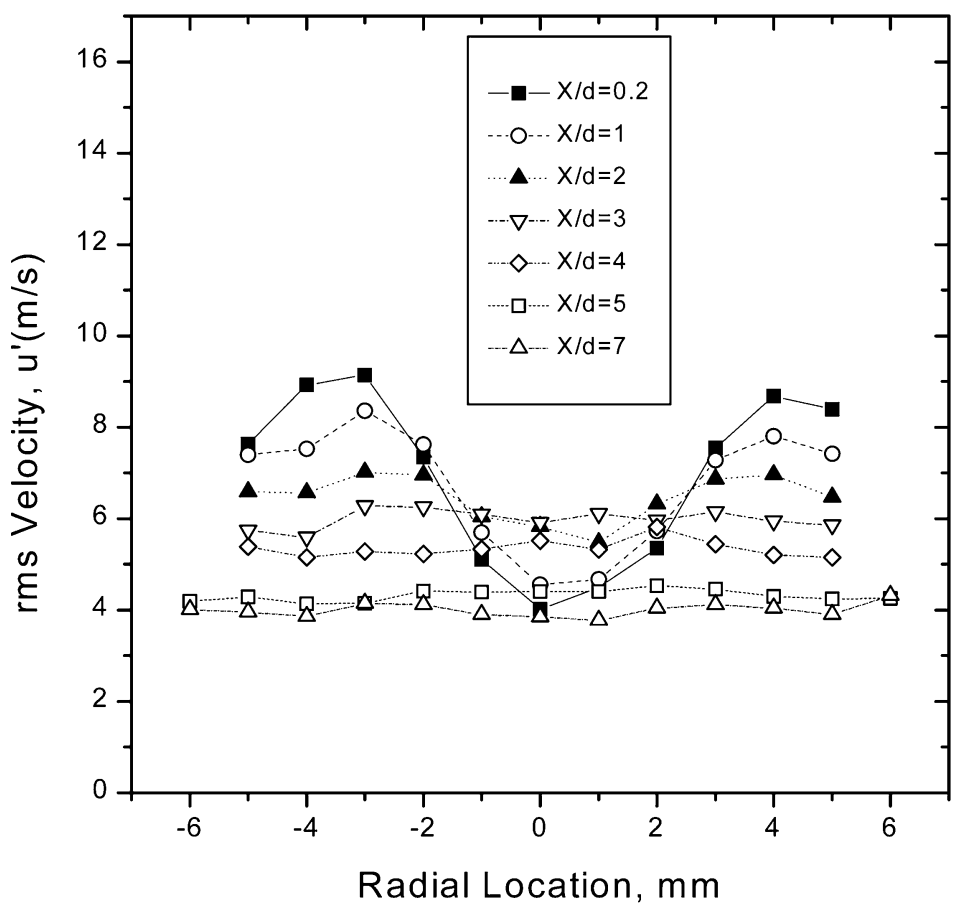

Figure 1. Turbulence intensity, $u^{\prime}$, profiles at various downstream locations for the flame set "M." $X$ is the axial distance along flame centerline and $d$ is the nozzle diameter.

pixel values of 0 indicate a progress variable of 0 (fresh mixture). The resulting image represents the instantaneous map of the progress variable. By averaging over the number of images selected (100 to 800) the mean progress variable map is determined. Individual flame contours (1 pixel wide) were detected from the instantaneous maps of the fresh/burned gases. Averaging the flame contours provides a map of the probability of presence of the flame front.

The approach for determining $\Sigma$ was derived from the formulation of Eq. (2). To evaluate $\Sigma(x)$, the instantaneous local two-dimensional flame surface density, $\Sigma_{x y}^{\prime}(x)$, needs to be corrected by the individual flame front orientation angle, $\theta$, observed in the orthogonal plane. $\Sigma_{x y}^{\prime}(x)$, which is the length of intersection between the flame front surface and plane $x y$, per unit area, was calculated from the product of the gradient $\left|\nabla c_{i}\right|_{x y}$ with the individual contour map. The orientation of the normal to the flame front, $\theta_{\mathbf{x y}}$, and $\Sigma_{x y}^{\prime}(x)$ were determined from individual maps of fresh/burned gases. 
As the Bunsen flame is axisymmetric, we obtained $\Sigma(x)$ by dividing $\Sigma_{x y}^{\prime}(x)$ by $\cos \left\langle\theta_{x y}\right\rangle$, which must be statistically equal to $\cos \left\langle\theta_{x z}\right\rangle$ along the axis of the burner assuming isotropic turbulence. Further details on the burner, LIF imaging system, image processing, and surface density determination may be found in Deschamps et al. (1996).

\section{RESULTS AND DISCUSSION}

A summary of results is given in Table 1 . The $\Sigma(x)$ profile, including individual data points, for one representative Bunsen flame is shown on Figure 2 as a function of reaction progress variable $\langle c\rangle$. This shows data averaged from 800 flame images. The solid line in Figure 2 represents a Lowess smooth of the data, and the maximum flame surface density is determined from this fit. The mean local two-dimensional flame surface density, $\Sigma_{x y}(x)$, and the mean orientation angle which produced the $\Sigma(x)$ profiles were also determined. The mean direction cosines $(\cos \langle\theta\rangle)$ of the flame front had a typical value of 0.69 for all the Bunsen flames. This is in good agreement with the typical value of 0.7 found in numerous studies (Trouvé and Poinsot, 1994; Deschamps et al., 1996; Shepherd and Ashurst, 1992).

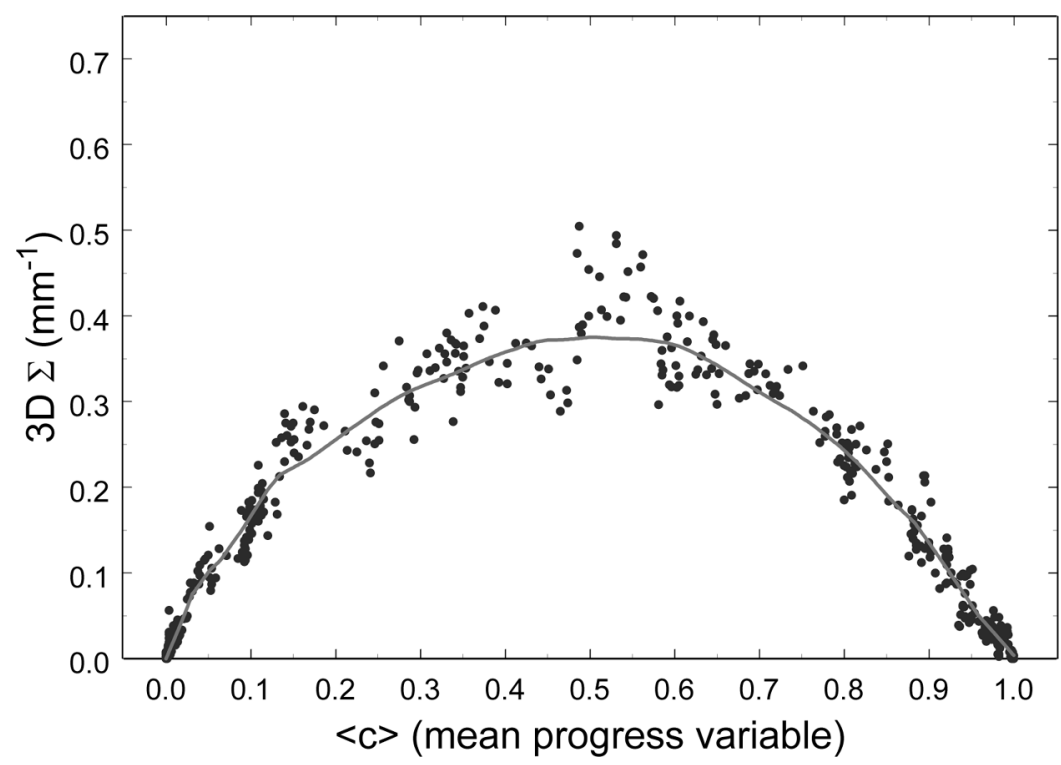

Figure 2. A typical flame surface density profile as a function mean progress variable, for flame set "E." 
The profile shown in Figure 2 is typical of those found for the other flames in this study. They are also typical of those found at low to moderate turbulence intensities $\left(u^{\prime} / S_{\mathrm{L}}=0.25\right.$ to 2.0$)$ in Bunsen flames and spark ignition engines (Deschamps et al., 1996). The profiles determined in this study are comparable in shape to those found by Veynante et al. (1994) in a two-dimensional V-flame, by Deschamps et al. (1994) in a Bunsen flame, and by Boger et al. (1998) obtained by direct numerical simulation. $\Sigma_{\max }$ has little variation over the range of $u^{\prime} / S_{\mathrm{L}}(0.84$ to 15.0) investigated here, as shown in Figure 3. Experimental flame surface density data reported in literature have also been included in Figure 3 for comparison. Clearly, there is no systematic relationship between the maximum flame surface density and the turbulence intensity. Results from direct numerical simulation efforts (Bell et al., 2002; Boger et al., 1998) also do not indicate any dependence of the flame surface density on turbulent rms velocity.

In Eq. (1), the mean rate of reaction, $\langle\dot{\omega}\rangle(x)$, and the flame surface density, $\Sigma(x)$, both vary with time and position; they are mean local

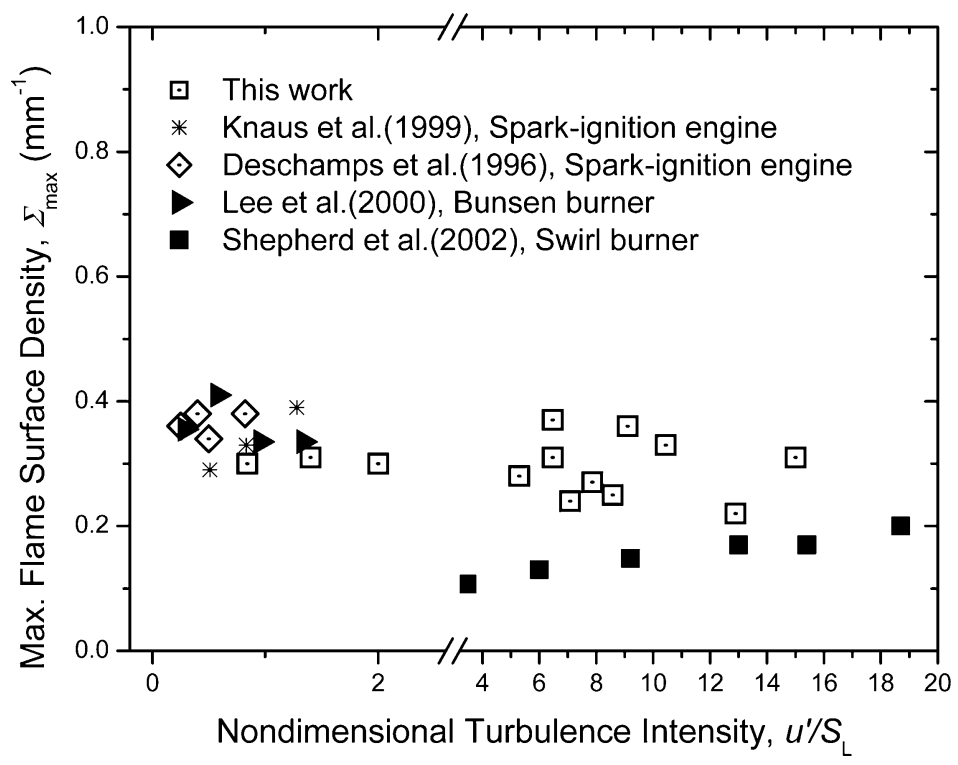

Figure 3. Maximum flame surface density versus non-dimensional turbulence intensity. Also shown are the results from Deschamps et al., 1996; Knaus et al., 1999; Lee et al., 2000; Shepherd et al., 2002. Note the axis break and scale change on the $x$-axis. 
properties. An overall heat release $Q$ within the turbulent flame brush of volume $V$ can be written as

$$
Q=\int \rho_{\mathrm{u}} S_{\mathrm{L}} \Delta H \Sigma \mathrm{d} V
$$

where $\rho_{\mathrm{u}}$ is the unburned mixture density, $S_{\mathrm{L}}$ is the laminar burning velocity, $\Sigma$ is the flame surface density, and $\Delta H$ is the enthalpy of reaction of the premixed mixture. For a well-defined geometry of a flame front, overall heat release can be also expressed in terms of a turbulent burning velocity $S_{\mathrm{T}}$ as:

$$
Q=\rho_{\mathrm{u}} S_{\mathrm{T}} \Delta H A_{\mathrm{o}}
$$

where $A_{\mathrm{o}}$ is the area perpendicular to the direction of the flame propagation. These expressions for $Q$ yield

$$
\frac{S_{\mathrm{T}}}{S_{\mathrm{L}}}=\frac{\int \Sigma \mathrm{d} V}{A_{\mathrm{o}}}
$$

Eq. (1) is equivalent to the Damköhler's hypothesis that

$$
\frac{S_{\mathrm{T}}}{S_{\mathrm{L}}}=\frac{A_{\mathrm{T}}}{A_{\mathrm{L}}}
$$

For practical purposes, the turbulent flame brush is commonly taken as the flame reaction zone bounded by the surfaces of $\langle c\rangle=0.05$ and $\langle c\rangle=0.95$. In view of Eq. (5), if we ignore the flame stretch and curvature effects for the moment, the turbulent burning velocity is a function of the integrated flame surface density. Integrated flame surface densities calculated from the flame images are shown in Figure 4 as a function of the non-dimensional turbulence intensity. Also shown in Figure 4 are the integrated flame surface density data reported by Chen and Bilger (2002) at low turbulence intensities obtained on Bunsen burner flames.

Integrated flame surface density shows no clear dependence on the turbulence intensity for the turbulent flame conditions studied in the present work. The observations that the integrated flame surface density do not change with the non-dimensional turbulence intensity have some serious implications. Experimental measurements on turbulent premixed flames have shown that the turbulent burning velocity increases with increasing turbulence. Table 1 shows the turbulent burning velocities estimated from the ratio of the area of an equivalent laminar cone ( $A_{\mathrm{L}}=q / S_{\mathrm{L}}$, where $q$ is the volume flow rate of the premixed reactants 


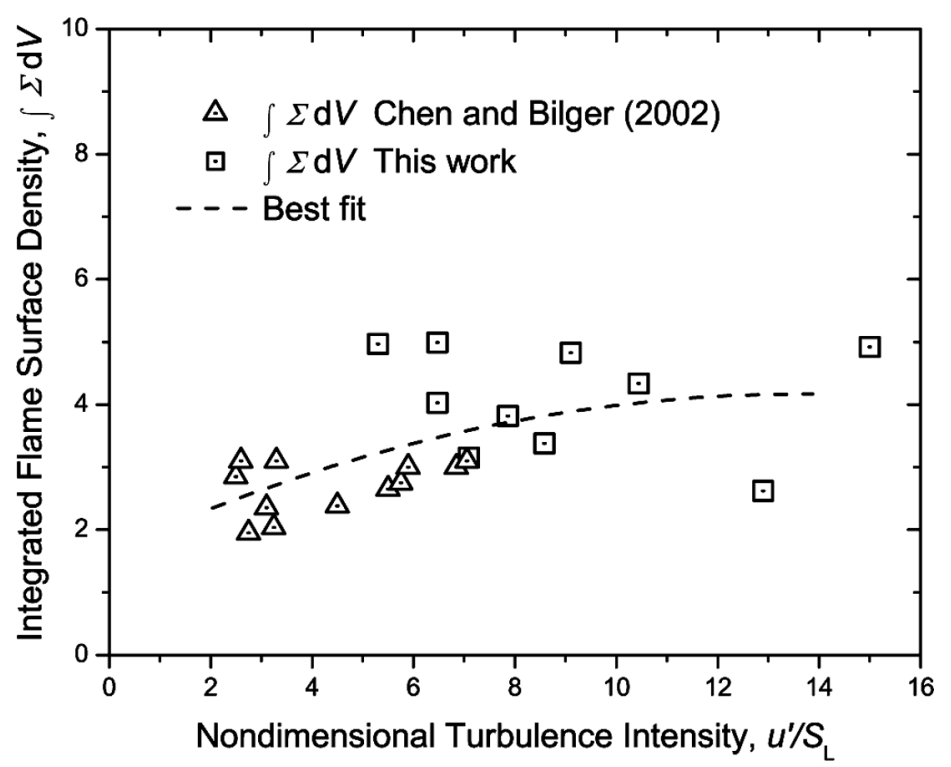

Figure 4. Integrated flame surface density data plotted as a function of nondimensional turbulence intensity. Also shown are the data from Chen and Bilger (2002). Dashed line indicates the best second-degree polynomial fit to the data.

and $A_{\mathrm{L}}$ is the area of the equivalent cone) to the area marked by the mean progress variable $\langle c\rangle=0.05$. The area marked by $\langle c\rangle=0.05$ denotes the lower bound of the flame brush. The turbulent burning velocity $S_{\mathrm{T}}$ increases as the turbulence intensity is increased. Thus the integrated flame surface density is expected to increase with increasing $u^{\prime} / S_{\mathrm{L}}$ in accordance with Eq. (5), however, it does not show any evidence of significant dependence on the flow turbulence.

If the integrated flame surface density at medium and high intensities is insensitive to turbulence parameters, then the fundamental assumption that the increasing turbulence intensity causes an increase in the flame surface wrinkling should be re-examined.

A second parameter in the closure model is the flamelet displacement or consumption velocity, $S_{\mathrm{d}}$. The magnitude of $S_{\mathrm{d}}$ as inferred from measurements and (direct) numerical simulations varies significantly and the relative influences of flame curvature and aerodynamic stretch seem to be unresolved yet. Recent evidence for the variation in $S_{\mathrm{d}}$ is summarized as follows. Sinibaldi et al. (1998) showed that the displacement 
velocity varies enormously along the flame, as much as a factor of 7.5 (from 0.7 to 5.25 times the unstretched value), and the flame curvature plays a dominant role in determining the displacement velocity. On the other hand, Peters et al. (1998) conclude that the displacement velocity is approximately $40 \%$ larger than the corresponding unstretched laminar burning velocity for a stoichiometric methane flame and $40 \%$ smaller for a lean flame. The strain rates that would quench the flame have been quantified by Bradley et al. (1998) and the results have been used to evaluate the $S_{\mathrm{d}} / S_{\mathrm{L}}$ ratio. More conservative approaches assume that for flames with Lewis numbers close to unity, $S_{\mathrm{d}}$ remains close to the laminar flame velocity (Trouvé and Poinsot, 1994; Veynante et al., 1997).

Neglecting the effects of $S_{\mathrm{d}}$ for the sake of discussion, assuming that it remains close to $S_{\mathrm{L}}$, then we are faced with three possibilities:

1. OH is not a good marker for flame front imaging and this is the cause of current controversial results: Laser-induced fluorescence of $\mathrm{OH}$ is one of the most commonly used flame front markers. Although a recent experimental effort implied that $\mathrm{OH}$ is not as good as the product of $\mathrm{OH}$ and $\mathrm{CH}_{2} \mathrm{O}$ as an indicator of heat release rate (Paul and Najm, 1998), it is adequate to mark the flame front location (Gülder et al., 2000).

2. The flames studied in the present work are not within the flamelet combustion regime (i.e., thin reaction zones regime): Figure 5 is a premixed turbulent combustion diagram. It shows the approximate domain of the flamelet combustion regime as redefined by Poinsot et al. (1991), Roberts et al. (1993), and Gülder and Smallwood (1995) in terms of the smallest eddies that could wrinkle the flame front. The most recent work of Peters (1999) also claims a wider domain for the flamelet regime as shown in Figure 5. The flames studied in the present work are plotted in Figure 5, and it is clear that they are within the flamelet combustion regime according to these most recent definitions.

3. The last but the most critical possibility, which follows from item 2 above, is that the flamelet combustion regime is much smaller and limited than presently believed. It may be confined to very low turbulence intensities of order $u^{\prime} \sim S_{\mathrm{L}}$ and large $\Lambda / \delta_{\mathrm{L}}$ ratios. This is in conflict with a number of theoretical and numerical results and calls for a reassessment of the assumptions leading to the current domain of the flamelet combustion on turbulent premixed combustion 


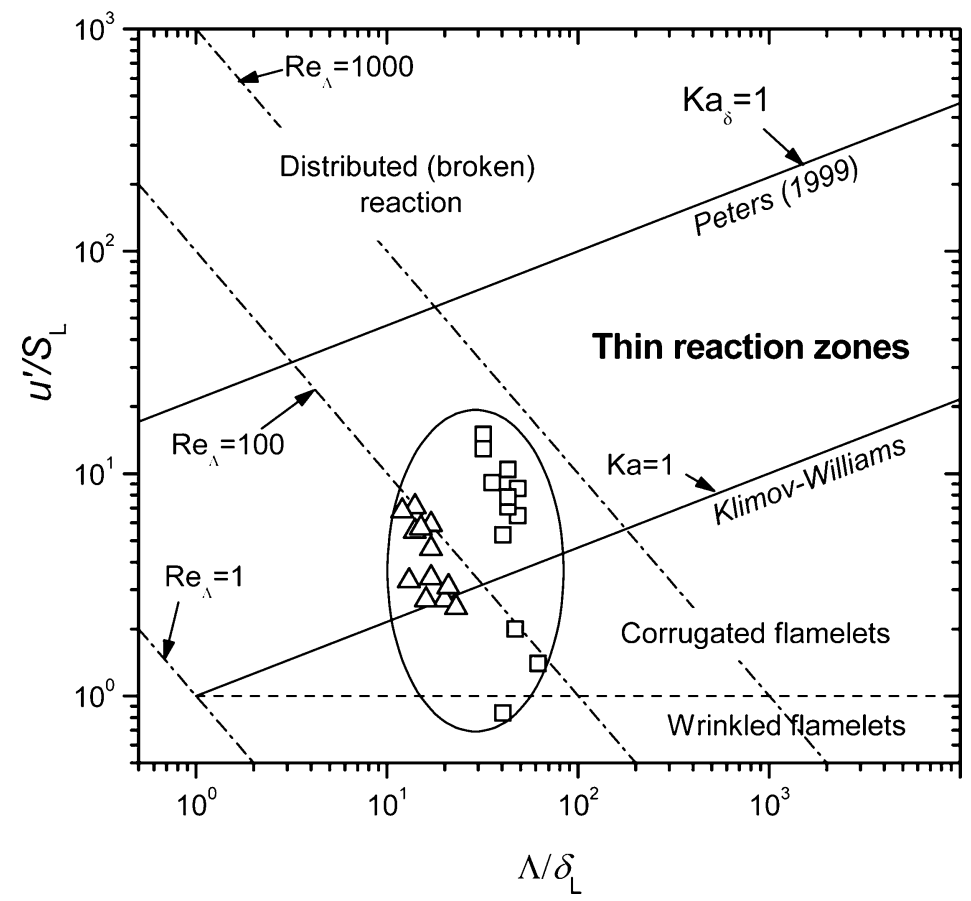

Figure 5. Diagram of premixed turbulent combustion regimes adapted from Peters (1999). Note the range of the thin reaction zone regime, and $\mathrm{Ka}_{\delta}=100 \mathrm{Ka}$. Circled area shows the two sets of data in Figure 4 (squares denote data reported in this work; triangles are from Chen and Bilger, 2002).

diagrams. Similar conclusions have been reached by applying the fractal geometry concept to premixed turbulent flames of Bunsentype burners (Gülder et al., 2000).

If the area increase (due to increasing turbulence) does not explain the increase in mean burning rate, then it may be proposed that the turbulent transfer of species and heat (enhanced by small-scale turbulence) should have a significant role in turbulent premixed combustion. The turbulent premixed combustion analysis and predictions should not be based only on the geometry of the flame front surface. This observation supports the analytical work of Ronney and Yakhot (1992) that concludes that the effect of scales smaller than the laminar flame front thickness are probably significant for most flames at sufficiently high turbulence intensities. It should be noted that thickening of the flame front by small-scale 
turbulence and its contribution to turbulent flame propagation were also proposed by Zimont (1979). The probability of the flame front alteration in the thin reaction zones regime by penetration of smaller size eddies into the flame front, and the enhancement of heat and mass transport should not be ignored, and there is some experimental evidence of this happening (Kortschik et al., 2004), although no direct experimental evidence of flame front thickenening has been reported.

\section{CONCLUDING REMARKS}

The characteristics of the flame surface densities in turbulent premixed propane/air flames were investigated using laser induced fluorescence of $\mathrm{OH}$ on two Bunsen type burners of 11.2 and $22.4 \mathrm{~mm}$ diameters in the nondimensional turbulence intensity $u^{\prime} / S_{\mathrm{L}}$ range from 0.84 to 15 . The Reynolds number based on the integral length scale was varied from 34 to 467 . These flames are in the flamelet combustion regime (in corrugated and in the thin reaction zones) as defined by the most recent turbulent premixed combustion diagrams.

In the non-dimensional turbulence intensity range of up to 15 , it was found that the maximum flame surface density and surface density profile as a function of the progress variable do not show any dependence on turbulence intensity. Also for the same flames, the integrated flame surface density was found to be insensitive to turbulence intensity.

Our findings imply that the conceptual increase in flame surface density by turbulence may not be the dominant mechanism for flame velocity enhancement in turbulent combustion in the region specified as the flamelet combustion regime by the current turbulent premixed combustion diagrams. Small-scale transport of heat and species may be more important and chemistry may not be decoupled from turbulence. Further, the applicability of the flamelet approach may be limited to a much smaller area than presently believed.

\section{ACKNOWLEDGMENTS}

We acknowledge the invaluable contributions of our colleagues, D. R. Snelling, Y. Côté, T. Zakutney, B. C. Benning and R. Smith. The work at National Research Council Canada was supported by the Canadian Government's PERD Program, and the work at University of Toronto by an NSERC-Collaborative Research Opportunities Grant (CRO). 


\section{REFERENCES}

Bédat, B. and Cheng, R.K. (1995) Experimental study of premixed flames in intense isotropic turbulence. Combust. Flame, 100, 485.

Bell, J.B., Day, M.S., and Grcar, J.F. (2002) Numerical simulation of premixed turbulent methane combustion. Proc. Combust. Instit., 29, 1987.

Bingham, D.C., Gouldin, F.C., and Knaus, D.A. (1998) Crossed-plane laser tomography: Direct measurement of the flamelet surface normal. Proc. Combust. Instit., 27, 77.

Bradley, D., Gaskell, P.H., and Gu, X.J. (1998) The modeling of aerodynamic strain rate and flame curvature effects in premixed turbulent combustion. Proc. Combust. Instit., 27, 849.

Bray, K.N.C. (1980) Turbulent flows with premixed reactants. In Libby, P.A. and Williams, F.A. (Eds.) Turbulent Reacting Flows, Springer-Verlag, p. 115-183.

Bray, K.N.C., Libby, P.A., and Moss, J.B. (1985) Unified modeling approach for premixed turbulent combustion 1. General formulation. Combust. Flame, $61,87$.

Bray, K.N.C. (1990) Studies of turbulent burning velocity. Proc. R. Soc. London. A431, 315.

Bray, K.N.C. and Cant, R.S. (1991) Some applications of Kolmogorov turbulence research in the field of combustion. Proc. Royal Soc. London Series A. 434, 217.

Boger, M., Veynante, D., Boughanem, H. and Trouvé, A. (1998) Direct numerical simulation analysis of flame surface density concept for large eddy simulation of turbulent premixed combustion. Proc. Combust. Instit., 27, 917.

Cant, R.S. and Bray, K.N.C. (1988) Strained laminar flamelet calculations of premixed turbulent combustion in a closed vessel. Proc. Combust. Instit., 22, 791.

Chen, Y.C. and Bilger, R.W. (2002) Experimental investigation of three-dimensional flame-front structure in premixed turbulent combustion-I: Hydrocarbon/air Bunsen flames. Combust. Flame, 131, 400.

Deschamps, B., Boukhalfa, A., Chauveau, C., Gökalp, I., Shepherd, I.G., and Cheng, R.K. (1994) An experimental estimation of flame surface density and mean reaction rate in turbulent premixed flames. Proc. Combust. Instit., 24, 469.

Deschamps, B.M., Smallwood, G.J., Prieur, J., Snelling, D.R., and Gülder, Ö.L. (1996) Surface density measurements of turbulent premixed flames in a spark-ignition engine and a Bunsen-type burner using planar laser-induced fluorescence. Proc. Combust. Instit., 26, 427.

Ghenaï, C., Chauveau, C., and Gökalp, I. (1996) Spatial and temporal dynamics of flamelets in turbulent premixed flames, Proc. Combust. Instit., 26, 331.

Gülder, Ö.L. and Smallwood, G.J. (1995) Inner cutoff scale of flame surface wrinkling in turbulent premixed flames. Combust. Flame, 103, 107. 
Gülder, Ö.L., Smallwood, G.J., Wong, R., Snelling, D.R., Smith, R., Deschamps, B.M., and Sautet, J.-C. (2000) Flame front surface characteristics in turbulent premixed propane/air combustion. Combust. Flame, 120, 407.

Knaus, D., Gouldin, F.C., Hinze, P.C., and Miles, P.C. (1999) Measurements of instantaneous flamelet surface normals and burning rate in a SI engine. SAE Paper No. 1999-01-3543, Society of Automotive Engineers, Warrendale, PA.

Kortschik, C., Plessing, T., and Peters, N. (2004) Laser optical investigation of turbulent transport of temperature ahead of the preheat zone in a premixed flame. Combust. Flame, 136, 43.

Lee, G.G., Huh, K.Y., and Kobayashi, H. (2000) Measurement and analysis of flame surface density for turbulent premixed combustion on a nozzle-type burner. Combust. Flame, 122, 43.

Marble, F.E. and Broadwell, J.E. (1977) The Coherent Flame Model for Turbulent Chemical Reactions. Project Squid Tech. Rep. TRW-9-PU, El Secundo, CA.

Paul, P.H. and Najm, H.N. (1998) Planar laser-induced fluorescence imaging of flame heat release rate. Proc. Combust. Instit., 27, 43.

Peters, N., Terhoeven, P., Chen, J.C., and Echekki, T. (1998) Statistics of flame displacement speeds from computations of 2-D unsteady methane-air flames. Proc. Combust. Instit., 27, 833.

Peters, N. (1999) The turbulent burning velocity for large-scale and small-scale turbulence. J. Fluid Mech., 384, 107.

Poinsot, T., Veynante, D., and Candel, S. (1990) Diagrams of premixed turbulent combustion based on direct numerical simulation. Proc. Combust. Instit., 23, 613.

Poinsot, T., Veynante, D., and Candel, S. (1991) Quenching processes and premixed turbulent combustion diagrams. J. Fluid Mech., 228, 561.

Pope, S.B. (1988) The evolution of surfaces in turbulence. Intl. J. Enginering Sci., 26, 445 .

Prasad, R.O.S., Paul, R.N., Sivathanu, Y.R., and Gore, J.P. (1999) An evaluation of combined flame surface density and mixture fraction models for nonisenthalpic premixed turbulent flames. Combust. Flame, 117, 514.

Roberts, W.L. Driscoll, J.F., Drake, M.C., and Goss, L.P. (1993) Images of the quenching of a flame by a vortex to quantify regimes of turbulent combustion. Combust. Flame, 94, 58.

Ronney, P.D. and Yakhot, V. (1992) Flame broadening effects on premixed turbulent flame speed. Combust. Sci. Technol., 86, 31.

Shepherd, I.G. and Ashurst, W.T. (1992) Flame front geometry in premixed turbulent flames. Proc. Combust. Instit., 24, 485.

Shepherd, I.G. (1996) Flame surface density and burning rate in premixed turbulent flames. Proc. Combust. Instit., 26, 373.

Shepherd, I.G., Cheng, R.K., Plessing, T., Kortschik, C., and Peters, N. (2002) Premixed flame front structure in intense turbulence. Proc. Combust. Instit., 29, 1833. 
Sinibaldi, J.O., Mueller, C.J., and Driscoll, J.F. (1998) Local flame propagation speed along wrinkled, unsteady, stretched premixed flames. Proc. Combust. Instit., 27, 827.

Smallwood, G.J. and Deschamps, B.M. (1996) Flame surface density measurements with PLIF in an SI engine. SAE Paper No. 962088, Society of Automotive Engineers, Warrendale, PA.

Trouvé, A. and Poinsot, T. (1994) The evolution equation for the flame-surface density in turbulent premixed combustion. J. Fluid Mech., 278, 1.

Veynante, D., Duclos, J.M., and Piana, J. (1994) Experimental analysis of flamelet models for premixed turbulent combustion. Proc. Combust. Instit., 25, 1249.

Veynante, D., Trouvé, A., Bray, K.N.C., and Mantel, T. (1997) Gradient and counter-gradient scalar transport in turbulent premixed flames. J. Fluid Mech., 332, 263.

Zhang, Y., Bray, K.N.C., and Rogg, B. (1998) The odeling and measurement of local flame surface orientation in turbulent premixed flames. Combust. Sci. Technol., 137, 347.

Zimont, V.L. (1979) Theory of turbulent combustion of a homogeneous fuel mixture at high Reynolds numbers. Comb. Expl. Shock Waves, 15, 305.

Zimont, V.L. (2000) Gas premixed combustion at high turbulence. Turbulent flame closure combustion model. Experiment. Thermal Fluid Sci., 21, 179. 